\title{
Transforming a surgical department during the outbreak of new coronavirus pandemic. Clinical implications
}

\author{
Ramon Vilallonga ${ }^{1}$ (1) - Amador Garcia Ruiz de Gordejuela ${ }^{1} \cdot$ Yolima Cossio-Gil ${ }^{2,3}$ • \\ José Manuel Domínguez González ${ }^{4} \cdot$ Rocio Martín Sánchez ${ }^{5} \cdot$ Manel Armengol Carrasco $^{5}$
}

Received: 25 May 2020 / Accepted: 8 July 2020 / Published online: 6 August 2020

(C) Springer-Verlag GmbH Germany, part of Springer Nature 2020

\begin{abstract}
Background The 2019 novel coronavirus (2019-nCoV) has caused an outbreak of the disease now officially named coronavirus disease 2019 (COVID-19). Since then, all hospitals have required a complete restructuring of their usual facilities and the treatments provided. Our goal was to detail the remodeling of a tertiary hospital during the COVID-19 outbreak and analyze pitfalls to avoid increasing surgical department burdens.

Methods This was a retrospective analysis of data affecting patients during their admission in our institution during March 2020. Data from general admission, intensive care units, and elective and emergency surgeries were collected and analyzed. All patients who underwent a surgical procedure were reviewed to elucidate limitations in the deployment of the hospital transformation to a COVID-19 hospital.

Results A total of 688 patients have been treated in our institution. Of those, 186 required intensive care. More than 120 new intensive care beds have been created during this period, and a decrease in elective surgeries of more than $75 \%$ was observed. Inadvertent COVID-19 patients accounted for 70\%. Thirty percent of the patients who underwent surgery while infected with COVID-19 died in our institution.

Conclusions The complete reorganization of surgical departments will be requested during the outbreak and adaptive solutions are needed in order to avoid increased mortality rates and infection among patients and to promote maximal optimization of surgical spaces. Timing, governmental decisions, and scientific society's recommendations may be limitations in the efficient deployment of hospital transformations to COVID-19 facilities.
\end{abstract}

Keywords Severe acute respiratory syndrome-coronavirus- $2 \cdot$ COVID-19 $\cdot$ Pneumonia $\cdot$ Hospital remodeling

Ramon Vilallonga

vilallongapuy@gmail.com

1 Endocrine, Metabolic and Bariatric Unit, Center of Excellence for the EAC-BC, Vall d'Hebron University Hospital, Universitat Autònoma de Barcelona, Passeig de la Vall d'Hebron 119-129, 08035 Barcelona, Spain

2 Department of information Systems and decision support, Hospital Universitari Vall d'Hebron, Barcelona, Spain

3 Healthcare Systems Research Group, VHIR, Universitat Autònoma de Barcelona, Passeig de la Vall d'Hebron 119-129, 08035 Barcelona, Spain

4 Vascular Surgery Department, Vall d'Hebron University Hospital, Universitat Autònoma de Barcelona, Passeig de la Vall d'Hebron 119-129, 08035 Barcelona, Spain

5 General Surgery Department, Vall d'Hebron University Hospital, Universitat Autònoma de Barcelona, Passeig de la Vall d'Hebron 119-129, 08035 Barcelona, Spain

\section{Introduction}

The first pneumonia cases of unknown origin were identified in Wuhan, China, in early December 2019 [1]. This causative pathogen has now been identified as severe acute respiratory syndrome-coronavirus-2 (SARS-CoV-2). The World Health Organization (WHO) has declared the novel coronavirus (COVID-19) disease as an international public health emergency. As of April 1, 2020, the total number of cases worldwide is 886,$278 ; 102,136$ of which are in Spain and have resulted in a total of 9053 deaths $[2,3]$.

During this challenging period, it is very important to give a comprehensive view of the situation in conjunction with the analyses done by epidemiologists to understand the pandemic situation [4]. Given the fast spreading nature of this virus, the lack of a fast and effective response from scientific community and government agencies might increase the number of 
patients affected by SARS-CoV-2. As we saw in China, COVID-19 affects a significant number of people, 30-40\% of whom will require hospitalization, and up to $10 \%$ may need also need observation in an intensive care unit (ICU). The time of admission varies between 6 and 7 days to 3-4 weeks [5]; thus hospital capacities and equipment availability may be overwhelmed. This situation could reshape the procedural norms of any hospital, including a necessity to completely reorganize and reallocate the types of beds available (i.e., intensive care beds vs. specialty wards). Additionally, doctors from various specialties may have to be redeployed to provide support for COVID-19 specialists.

Data suggests that some patients are asymptomatic; thus, undetected COVID-19 may lead to contamination of wards, operation rooms, equipment, and even healthcare professionals [6]. Management of these patients is particularly challenging, both for the high dissemination capacity of the virus and the severity of disease in some patients. Handling of surgical patients is even more challenging in the following aspects: First, increased staff exposure and therefore needed protection throughout pre-, peri-, and post-surgery procedures; second, the patient's COVID-19-related morbidity being potentiated by the surgical procedure [7].

To aid this necessary metamorphosis, surgical wards, surgical lists, and surgical rooms have completely modified their conventional roles and rules. The aim of this study is to analyze and describe the impact of the COVID-19 pandemic on hospital procedures, specifically in an adult surgery department in a tertiary care hospital, including a perspective on the management of surgical COVID-19 patients in the emergency room.

\section{Methods}

\section{Study design}

This was a retrospective analysis using a daily database for information and decision-making. We analyzed data available from our institution published from March 1 to April 1, 2020, which includes hospital-wide data using cited methodology.

\section{Settings}

Our institution is a tertiary hospital in Spain, containing 1100 patient beds. Throughout the hospital, there are 47 training programs and over 530 residents across the 22 buildings that make up the hospital. The Information systems are available to all teams including the epidemiologists that analyze, on a daily basis, any information that will allow better decisionmaking during the COVID-19 pandemic. The surgical department is the largest specialty within our system, geared toward serving a wide spectrum of diseases. Our institution is divided into four different buildings: the orthopedics hospital, the maternity and pediatrics hospital (pediatrics, gynecology, and obstetrics), the general hospital (serving mainly adults), and a satellite facility where ambulatory surgery is performed. Surgical departments from the general hospital were analyzed. Those included for the present study are urology, vascular surgery, cardiothoracic surgery, general surgery, pancreatic and hepatobiliary surgery (including liver transplant), otorhinolaryngology, thoracic surgery, and neurosurgery. Departments such as gynecology, orthopedics, plastic surgery, maxillofacial surgery, and pediatric surgery were excluded from this study as their daily activities were restricted to their respective buildings.

\section{Performance of the hospital and of the surgical department}

Performance of the hospital was analyzed by measuring the number of beds transformed to COVID-19 beds and the number of patient admissions and discharges related to COVID19. For the surgical procedures, performance was measured by the number of cases attended in the general hospital, including elective (scheduled) and emergency surgery. Comparative data extracted from the overall number of surgeries performed during the month of March were compared with surgeries performed in March of 2019. This data has been analyzed according to our general database and all the team leading information resources and decision-making. Elective surgery was discontinued on the 12th of March and only urgent cases were treated. All elective oncological and non-oncological cases were canceled. All elective and emergency patients were triaged in the waiting list according to their pathology and were postponed. Unfortunately, no specific surgical treatment was offered, and telephonic follow-up was done and acting accordingly.

\section{Surgical protocols}

During March 2020, data from surgical patients were classified as either scheduled or emergent procedures. A series of four updated protocols, aimed at minimizing contagions, were established in our center during the period of this study. The prioritized populations were confirmed patients infected with COVID-19, suspected cases of COVID-19, or those who had been in contact with infected individuals. Detection of the virus in these individuals was facilitated by using Reverse transcriptase-polymerase chain reaction (RT-PCR). Starting March 14, 2020, all scheduled surgeries were suspended since a "State of Alarm" was declared in Spain [8]. Two independent hubs, with few elective or urgent surgeries remaining, were reorganized; one to be used for patients who tested positive for COVID-19 patients and the other for those who tested negative for COVID-19. Surgical staff and operating rooms for infected patients were limited. All patients who underwent 
a procedure were screened for COVID-19 after March 16, 2020, and we identified if the diagnosis had been done before, or after surgery. For identified COVID-19 patients who underwent surgery during this period, we analyzed the clinical history, medical data, and surgical data available. Laboratory data for all patients included blood cell count and ferritin, interleukin-6 (IL6), and C-reactive protein (CRP) levels. Final outcome was included in the results for all patients.

Data are presented as number of cases recorded treated in our hospital during the study period and the mean and range for continuous variables, and number of cases and their percentages for quantitative variables. No comparisons were made with the data.

\section{Results}

During the analyzed period, we observed a complete reorganization of the hospital. The number of COVID-19 patients grew exponentially as is shown in Fig. 1 and the number of surgical procedures were reduced to a minimal level.

\section{Hospital capacity}

Throughout the hospital, a total of 1152 beds were available before the pandemic, of which 113 were for the ICU. In March 2020, the number of conventional and ICU beds increased or were assigned for COVID-19 patients. For the general hospital, there were 617 beds (56 ICU). During the study period, the number of ICU beds increased to 118 . The number of conventional beds also increased, and $89 \%$ of all of them were assigned to COVID-19 patients (Fig. 2).

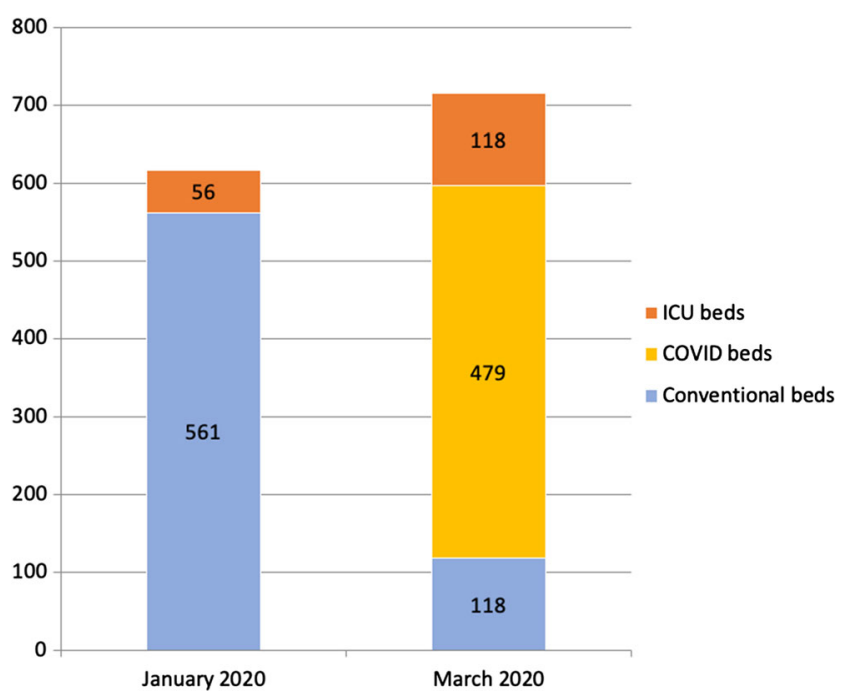

Fig. 1 Number of patients admitted into the ward or ICU including discharged patients and deaths: Prevalence cut per day in all of the hospital
Patients admitted in the general hospital building for COVID-19 during March 2020: A total of 2320 patients were admitted in the general hospital in March 2020, of whom 688 were COVID-19 positive. Of those, 653 were discharged, and 186 were admitted in the ICU. Unfortunately, 106 patients did not respond to treatment and died. Patient outcomes are summarized in Table 1.

\section{Surgical patients treated}

Data show a complete decrease in the number of patients treated for surgical diseases requiring a scheduled or emergency surgery during March 2020. We also observed a decrease in the number of procedures, compared with 2019 (Fig. 3). In 2019, a total of 719, versus 535 in 2020, underwent a major surgical procedure in the general hospital (excluding gynecology, pediatrics, and trauma). During the first 2 weeks of 2019 and 2020, total numbers of patients treated were 368 and 390, respectively. During the last 2 weeks of the month of March, the number of treated patients was 351 in 2019 and 145 in 2020. This decrease was more apparent after the second week when the total surgical procedures decreased $59 \%$, especially number of scheduled surgeries (reduction of $87 \%$ ), but also emergency procedures (reduction of $62 \%$ ). On March 16, 2020, surgical protocol for COVID-19 patients was implemented and included a complete regression of the elective surgery; after March 21, 2020, a double circuit for COVID-19 and non-COVID-19 patients was implemented in the surgical ward. Surgeons were not limited to work in COVID-19 and non-COVID-19 areas. Ten patients underwent a surgical procedure in the general hospital (excluding gynecology, pediatrics, and trauma), and their COVID-19 diagnosis was unknown. Diagnosis was suspected once in the hospitalization ward due to clinical symptoms and confirmed with CRP-COVID-19. All patients were then treated accordingly and isolated in a COVID-19 ward. Of those, 6 were emergency surgeries and were diagnosed before the procedure (Fig. 3). For all patients, clinical and laboratory data are reported in Table 2.

\section{Discussion}

Since December 2019, pneumonia caused by a novel coronavirus infection was identified in Wuhan City, in the Hubei Province of China [9]. Several provinces, autonomous regions, and municipalities have also reported confirmed and suspected cases. The World Health Organization (WHO) named this pathogenic virus 2019-nCoV [10]. In view of the general susceptibility of its population, on January 20, 2020, the National Health and Health Commission of the People's 


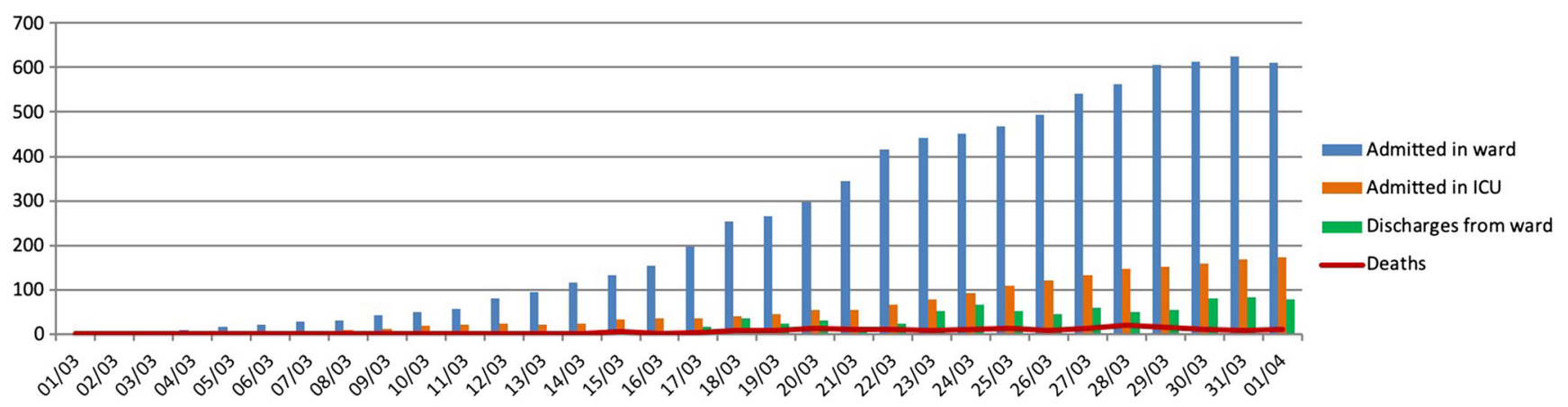

Fig. 2 Comparison of the number of beds in the General Hospital increased or assigned to COVID-19 patients before and during the period of study

Republic of China included pneumonia caused by new coronavirus infection into Class $\mathrm{B}$ infectious diseases and managed them according to Class A infectious diseases [10]. Under this policy, hospitals could then adopt isolation treatment and observation protocols to prevent and control the spread of the COVID-19. Initially, western countries were aware of the virus and only observed what was happening in China; however, no public health emergencies were declared nor were any preventative or control measures implemented. The first case reported in Spain was in Tenerife on February 27, 2020 [11]. On March 1, 2020, according to the Ministry of Health only 84 cases were confirmed in Spain and there was only one death reported [5]. After that, the hospital administration designed a contingency plan where they calculated different based on the behavior of the pandemic. These scenarios considered potential restrictions to surgical scheduling and defined protocols for surgical procedures in patients suspected or confirmed of infection status. A double COVID-19 and non-COVID-19 pathways was created since March 24. In the de-escalation of the pandemic, elective surgery has been included and preoperative work-up includes nowadays epidemiological history, PCR-COVID-19 $48 \mathrm{~h}$ before surgery. There is a need to protect patients and hospital staff during the reintroduction of the elective surgery. In emergency surgery, fast- RT-PCR-COVID-19 is requested and results are available in $1 \mathrm{~h}$.

Table 1 Total number of COVID-19 and non-COVID-19 patients admitted, discharged, and deceased in the general adult hospital during March 2020

\begin{tabular}{llll}
\hline & $\begin{array}{l}\text { COVID- } \\
19\end{array}$ & NO COVID-19 & Total \\
\hline Admitted in ward & 502 & 1439 & 1941 \\
Admitted in ICU & 186 & 193 & 379 \\
Total admitted in general hospital & 688 & 1632 & 2320 \\
Discharges from ward & 547 & 1487 & 2034 \\
Deaths in wards & 106 & 90 & 196 \\
Total discharges in general hospital & 653 & 1577 & 2230 \\
\hline
\end{tabular}

$I C U$ intensive care unit

\section{Building adaptation and reduction of the surgical activity}

Regarding the COVID-19 pandemic, it is very important to adopt measures to fight the pandemic through governmental policies and scientific expertise. All states should be well informed of the epidemiology of the virus in order take the appropriate course of action to fight the pandemic and know when to implement severe measures of prevention and testing. Our data clearly show that there is an overlap between viral spread, the time to show symptoms, and when COVID-19 patients appear in the emergency department (ED) and ICU [12]. During the pandemic growth, our institution increased the number of beds for COVID-19 patients by more than $400 \%$. This increase has seriously affected the ability to perform elective surgeries. Our data show a necessity for specified spaces for different types of patients. In fact, as we can see in our results, the number of COVID-19 patients is negatively correlated with those requiring "standard" treatments. A huge effort has to be made in the earlier phase of the pandemic and the whole hospital should be working in a same direction. Added effort should be made during the early stages of the pandemic, when patients and healthcare professionals are less physically and emotionally burdened. As we can see in our results, the number of beds dedicated to surgical patients decreased every week, and based on anecdotal reports, this was observed throughout the whole institution. In order to prevent chaos, new spaces were prepared for the patient influx. A similar experience was reported in Italy [13]. The peak of the pandemic was reached on March 31, 2020, in our Institution. At that time, elective and emergency surgery represented less than $5 \%$ of the procedures that entire month. The contingency plan, designed from the beginning as a progressive reduction in surgical procedures, approved only those procedures that could not postponed. Once the outbreak arrived, all scheduled procedures were canceled. Up to $90 \%$ of the wards were reassigned to deal with COVID-19-related matters. Doctors from different specialties (surgical and nonsurgical) were reassigned to work on multidisciplinary teams commanded by infectious disease, internal medicine, or pneumology specialists. Specific training was adopted in 


\section{Surgical Cases March 2020}

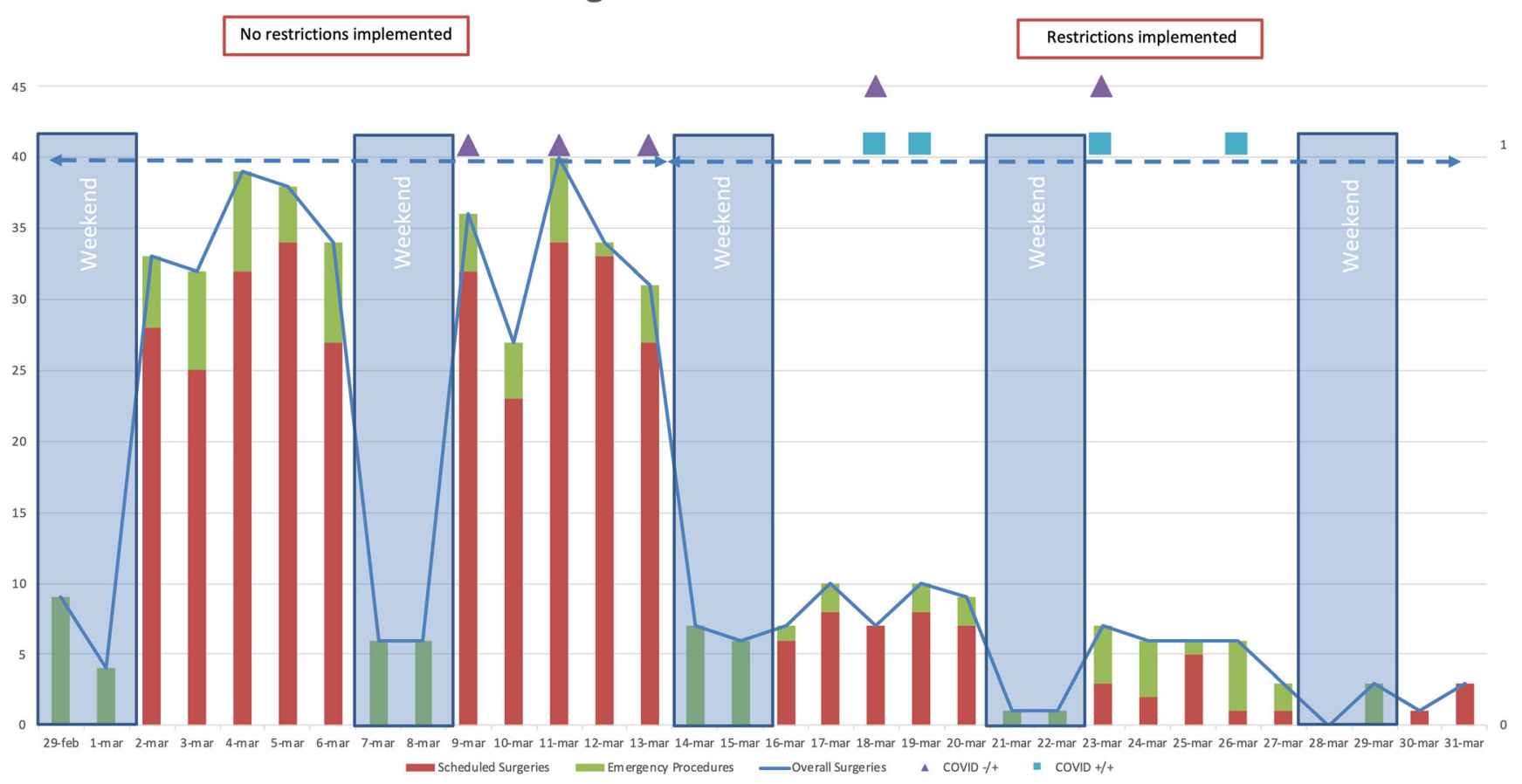

Fig. 3 Figure showing the number of patients treated in the general hospital during the month of March 2020. Emergency and elective surgeries are represented during the complete period. Patients who were diagnosed with COVID-19 are also represented

order to introduce protective protocols for personnel. Decision-making to endure the safety of the patients and healthcare professionals during this time was very challenging. Measures were implemented according to national guidelines [14] in order to redistribute the beds for COVID-19 patients while also protecting patients with and without COVID19 from personnel and vice versa.

\section{Management of surgical pathologies during the pandemic}

A "war-medicine" strategy must be implemented in this scenario. Scheduled surgery was not available during the pandemic, but surgical patients needed to be managed. As such, different international societies began to write protocols and guidelines $[15$, 16]. There are no clear recommendations because this experience is unprecedented. Data suggests that oncological patients should be prioritized [16, 17]. Literature regarding the effects of remodeling surgical departments, including the non-operated elective surgeries postponed during the COVID-19 pandemic, is still not available. However, intuitive data and personal experience will show that patient care is more inefficient after being left untreated; The impact of having a longer time before elective surgery in some cases will affect the overall prognosis and technical (surgical) requirements. This can be due to a worsened prognosis of the tumor for example (in case of oncological pathology) or because the patient is admitted for necessity of an emergency surgery rather than a planned elective surgery.
Many surgical societies are now recommending postponing elective surgical treatments for the time being and rescheduling them after the pandemic is under control. For patients with limited-term surgery, the timing of surgery can be delayed appropriately without affecting the condition of the patient or treatment outcomes; for patients with intermediate and advanced malignancies, after discussion within multidisciplinary team, if the neoadjuvant treatment is expected to be effective, fully communicate this with the patient's family. This situation is very common in colorectal surgery [18]. For oncological patients, different patterns of chemotherapy have been implemented as a surgical substitute. Patients with non-oncological emergencies should be evaluated on a case by case basis and advised about the risk of delaying the procedure vs. the risk contracting COVID-19 [19]. This equation is difficult to balance and should also consider hospital resources. Evidence from China suggests canceling all elective procedures and focusing on 2019-nCoV patients. The resources, both physical and human, are consumed by COVID19 thereby making it near impossible to efficiently make any reservations for other pathologies.

\section{Elective and emergency surgery during the COVID-19 pandemic era}

According to our experience, we have decreased to $60 \%$ of our planned activity in 1 month although having a normal program during the first 2 weeks of the month of March 2020 in which a normal activity was planned. How 


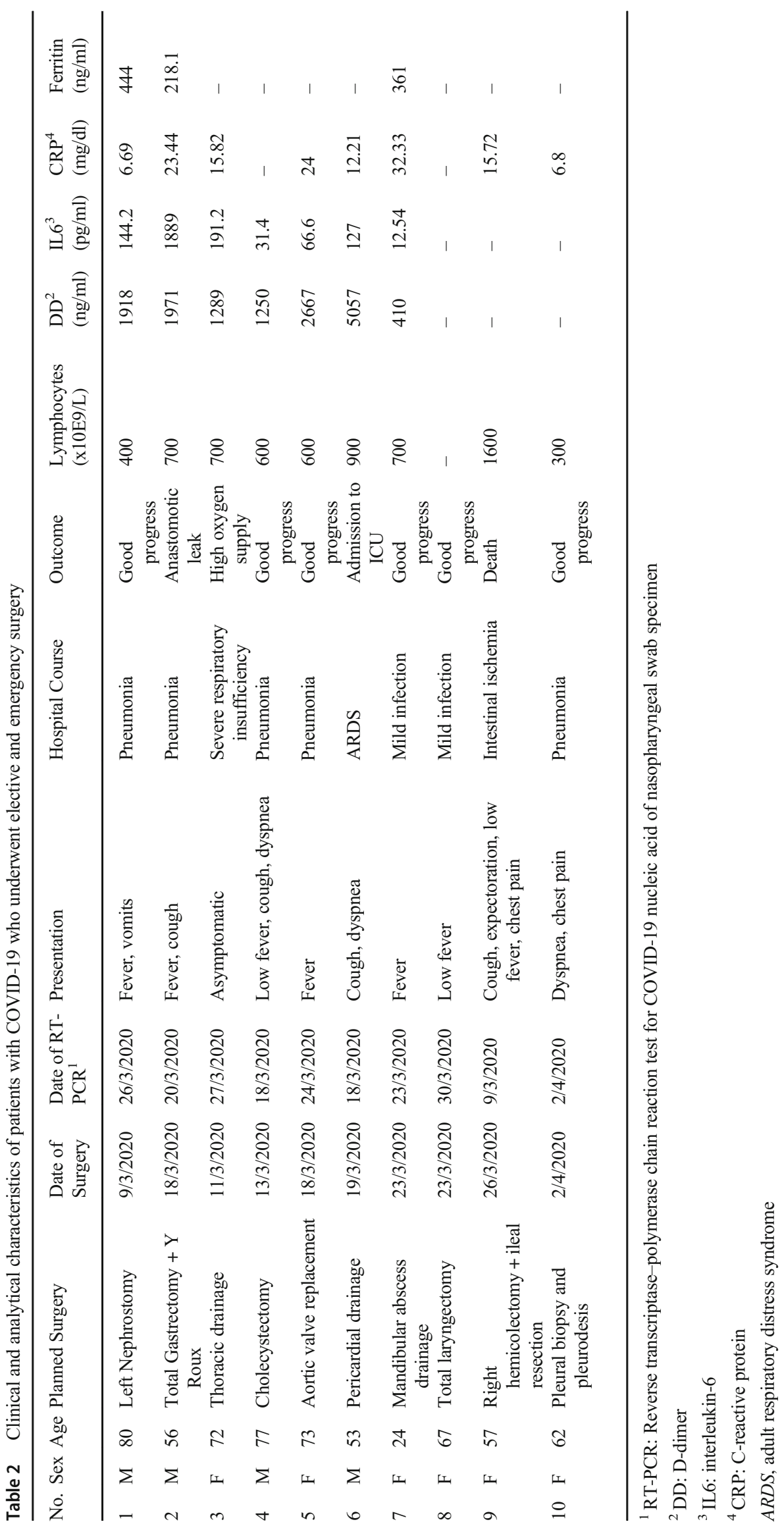


we deal with the co-existence of a patient's disease and COVID-19 is imperative. In our institution, we implemented screening for COVID-19 in patients after the third week, showing that still some patients were positive and were undiagnosed. Since the beginning, Thorax X-Ray was requested but lacked sensitivity to provide successful outcomes. Our results show five patients underwent emergency/elective surgery without having a diagnosis of COVID-19. We should recommend that RT-PCR testing should always be available to patients. Nevertheless, RT-PCR might have some latency that is not compatible with the emergency of the procedure. Quick tests may have a low sensitivity that leads to inconclusive results. Finally, most of the guidelines recommend a thoracic CT-scan to look for early sings of the disease. On the other hand, some surgeons propose to treat all surgical patients as positive for COVID-19. This strategy is the safest but is also very expensive in terms of resources especially in places where lack of personal protective equipment (PPE) has been an issue. In the present COVID-19 pandemic, there is concern among surgeons regarding the potential risk of viral transmission through the smoke or aerosols originated by electrocautery or energy devices (ultrasonic shears) during laparoscopic. Recently, more data is available regarding the need to use smoke evacuators, negative pressured OR, intubation, and extubate protocols to treat COVID-19 patients previously diagnosed requiring an elective/urgent surgery [20, 21]. Also, despite the recommendations of surgical associations and specialists in laparoscopy, such possibilities have not been ruled out, and COVID-19 RNA has been detected throughout the respiratory tract, in feces, in the intestinal mucosa, and in peritoneal fluid. [22, 23].

For emergency surgery patients, after suspicion of COVID19 has been eliminated, the operation can be performed according to normal surgical guidelines. Suspected or confirmed positive COVID-19 patients should be reported to the hospital management department and communicated to all related departments, such that surgical teams can proceed with strict precautions. Additionally, it is very important to understand the comorbidities associated with surgical procedures. In our short series of patients, two patients died from postoperative complications. Another patient who underwent gastric surgery experienced a leak that required treatment, thus considered a major complication. Some authors from Iran have already reported a higher incidence of postoperative complications after elective surgeries $[19,24]$. Other experiences have showed higher postoperative sensitivities in COVID-19 patients, including major postoperative complications $[25,26]$. Laboratory data do not seem to predict postoperative complications, so far. However, some studies are trying to relate inflammatory markers with pulmonary disease prognosis during the pneumonia [27]. Our data show that previous pneumonia or the development of pneumonia during the postoperative period can be a major medical complication for the patient and for the overall surgical process. Some data suggests that COVID-19 patients can experience increased complications before or after the surgery and this would lead to poor health outcomes. Regarding the final prognosis of the actual disease treated with surgery, some data suggest that patients with COVID-19 would have poor postoperative results and a worse recovery period after the surgery. Preventative measures must be taken during admission or in the postoperative ward in order to avoid the spread of COVID19 to patients after surgery, as occurred in 6 of our patients.

As shown in our results, emergency surgery was also reduced because of the quarantine dictated by the State on March 14, 2020, and probably potentiated by social insecurities concerning COVID-19. This aspect is very important because, as shown in our results, the number of surgical emergencies treated in the emergency room decreased dramatically before quarantine (9th to 15 th of March) and then continued to decrease after quarantine (after 16th of March).

This situation could potentially lead to different difficulties that management of entire surgical departments will need to face. First, the cases arriving in the ED will be of higher complexity. Patients may opt to stay at home longer rather than going to the ED. This will require optimization of patient care. Second, the COVID-19 pandemic and associated quarantine, and the lack of tests available for the population, will lead to an increase in the number of patients with COVID-19 that will eventually require treatment. This could explain the number of COVID-19 positive patients that underwent a procedure without testing. The pandemic has led us a critical situation and a need of training in an area for all personnel involved in the surgical management of patients. Time has been a pitfall and the lack of serological tests to protect surgeons, nurses, but also to better treat patients. Another pitfall we have detected has been the rhythm of novel decisions according to the daily state of the pandemic, coming from the Government and adapting to the hospital situation.

\section{Surgery department survival and future}

This paper shows how the decrease of the elective and emergency surgery has been necessary during the pandemic. Overall, the number of admitted patients shows a slow decrease since April 1, 2020. Once the pandemic passes, surgical services will have to be reestablished, including overcoming restrictions related to lack of wards or ICU and the evaluation of surgical waiting lists. Future data will probably show that the current pandemic has resulted in some collateral damage. For instance, some patients may have a worsening of their condition prior to their newly scheduled surgeries, some may experience more advanced tumors, and some patients will not ever be candidates for surgical treatment. These data will be extremely difficult to quantify; however, we will collect data as it becomes available and as restaging tumors will 
be needed in the next months. Another aspect in which we agreed was in the staff being kept on standby to return to the ad hoc roles when required. Also, the Institution included a de-escalation program to return the hospital to a "normal" non-COVID-19 situation. For the future, previous experience will be needed, and decision-making will be easier for all members of the institution. More recent available literature will help us to better understand the pandemic, its effects, and better approaches.

This study has both weaknesses and strengths. The biggest weakness is the relatively fast analysis of the data in a realtime emergency and also trying to include hospital and surgical adaptation of an institution. However, this study also represents a unique and early experience of a major tertiary hospital in Spain. Lessons learnt include faster serological test applied in any institution in order to treat and protect staff and patients, a continuous educational training in infections pandemic and an improved communication with other hospitals facing the same situation but earlier. Our main contribution is detailing the accomplishments and difficulties of transforming a hospital for COVID-19. Finally, COVID-19 is here to stay, thus we may face new epidemics in the near future. Protocols for early diagnosis and departments dedicated for those patients will be designed. In the future, we will need to consider the presence of antibodies or M2 in patients that may have already been infected by COVID-19. New protocols will be needed in order to face in better situations a new pandemic in the future.

\section{Conclusions}

This case series shows the challenges facing surgical practice in the initial phase of the 2019-nCoV outbreak. In our region, starting in early March, the incidence of 2019-nCoV grew exponentially, and following the contingency protocol, from March 10, 2020, onward, adaptations were initiated, and all surgical procedures were canceled. For 1 week, patients whose surgeries could not be postponed were scheduled in only 2 or 3 surgical rooms. Planning of the entire hospital, institutions, and buildings according to real-time data is necessary to optimize the efficiency of the institution and its careproviders. It is also very important to focus our attention on preventing the dissemination of all areas of the department and acting accordingly. Finally, the next months will tell us, according to the best evidence, the potential need of a dual system in our surgical departments.

Acknowledgments We would like to acknowledge Mrs. Montserrat Suárez Bernal for her technical support and data analysis needed to understand the hospital transformation with the Lean Methodology and the management team led by Dr. Antonio Roman.

Availability of data and material Available at our institution.
Authors' contributions Study conception and design: RV and YCG; Acquisition of data: RV, RM and YCG; Analysis and interpretation of data: RV, JMD, AG and YCG; Drafting of manuscript: RV and YCG; Critical revision of manuscript: all authors.

Funding information There are no sources of support to be reported from any of the following organizations: National Institutes of Health (NIH); Wellcome Trust; Howard Hughes Medical Institute (HHMI); and others, including no grants for doing the present work.

\section{Compliance with ethical standards}

Conflict of interest The authors declare that they have no conflict of interest.

Ethical approval All procedures performed in studies involving human participants were in accordance with the ethical standards of the institutional and/or national research committee and with the 1964 Helsinki declaration and its later amendments or comparable ethical standards.

\section{References}

1. Wang C, Horby PW, Hayden FG, Gao GF (2020) A novel coronavirus outbreak of global health concern. Lancet 395:470-473

2. World Health Organization. Coronavirus (COVID-19) events as they happen available from: https://www.who.int/emergencies/ diseases/novel-coronavirus-2019/events-as-they-happen. Accessed 11 April 2020

3. Woelfel R, Corman VM, Guggemos W, et al. (2020) Clinical presentation and virological assessment of hospitalized cases of coronavirus disease 2019 in a travel-associated transmission cluster. medRxiv.;2020.03.05.20030502

4. COVID-19 Map - Johns Hopkins Coronavirus Resource Center Available from: https://coronavirus.jhu.edu/map.html. Accessed 7 April 2020

5. Instituto de Salut Calos III. Report on confirmed COVID-19 cases in Spain COVID-19 Report No. 4. March 02, 2020. Available from: ht tps://www.isciii.es/QueHacemos/Servicios/ VigilanciaSaludPublicaRENAVE/EnfermedadesTransmisibles/ Documents/INFORMES/Informes COVID-19/Informe COVID19. $\mathrm{N}^{\mathrm{o}} 4$ 02marzo2020_ISCIII.pdf. Accessed 7 April 2020

6. Brat GA, Facs M, Hersey SP et al (2020) Protecting surgical teams during the COVID-19 outbreak: a narrative review and clinical considerations. Dtsch Arztebl 117(18):A-940-B-793

7. World Health Organization. Coronavirus disease 2019 Available from: https://www.who.int/emergencies/diseases/novelcoronavirus-2019. Accessed 7 April 2020

8. BOE.es - Documento BOE-A-2020-3692 Available from: https:// www.boe.es/diario_boe/txt.php?id=BOE-A-2020-3692. Accessed 7 April 2020

9. World Health Organization. Situation Report-24 Available from: https:/www.who.int/docs/default-source/coronaviruse/situationreports/20200213-sitrep-24-covid-19.pdf?sfvrsn=9a7406a4 4. Accessed 11 April 2020

10. Organization WH. Statement on the second meeting of the International Health Regulations (2005) Emergency Committee regarding the outbreak of novel coronavirus (2019-nCoV). Geneva, Switz 2005;1-6

11. First Case confirmed in Spain. Available from: https://www. diariomedico.com/medicina/medicina-preventiva/primer-casoconfirmado-de-coronavirus-en-espana.html. Accessed 7 April 2020 
12. Guan W, Ni Z, Hu Y, Liang WH, Ou CQ, He JX, Liu L, Shan H, Lei CL, Hui DSC, du B, Li LJ, Zeng G, Yuen KY, Chen RC, Tang CL, Wang T, Chen PY, Xiang J, Li SY, Wang JL, Liang ZJ, Peng YX, Wei L, Liu Y, Hu YH, Peng P, Wang JM, Liu JY, Chen Z, Li G, Zheng ZJ, Qiu SQ, Luo J, Ye CJ, Zhu SY, Zhong NS, China Medical Treatment Expert Group for Covid-19 (2020) Clinical characteristics of coronavirus disease 2019 in China. N Engl J Med 28:2020-1720. https://doi.org/10.1056/nejmoa2002032

13. Zangrillo A, Beretta L, Silvani P, et al (2020) Fast reshaping of intensive care unit facilities in a large metropolitan hospital in Milan, Italy: facing the COVID-19 pandemic emergency. Crit Care Resusc. Available from: http://www.ncbi.nlm.nih.gov/ pubmed/32227819. 2020. Accessed 11 April 2020

14. Balibrea JM, Badia JM, Rubio Pérez I, et al (2020) Manejo quirúrgico de pacientes con infección por COVID-19. Recomendaciones de la Asociación Española de Cirujanos. Cirugía Española. DOI: https://doi.org/10.1016/j.ciresp.2020.03. 001

15. SAGES and EAES Recommendations Regarding Surgical Response to COVID-19 Crisis - SAGES Available from: https:// www.sages.org/recommendations-surgical-response-covid-19/. Accessed 8 April 2020

16. Brindle M, Gawande A. Managing (2020)COVID-19 in surgical systems. Ann Surg. DOI: https://doi.org/10.1097/SLA. 0000000000003923

17. Aminian A, Safari S, Razeghian-Jahromi A et al (2020) COVID-19 outbreak and surgical practice. Ann Surg 2020:1

18. Di Saverio S, Pata F, Gallo G et al (2020) Coronavirus pandemic and Colorectal surgery: practical advice based on the Italian experience. Color Dis 31:2020. https://doi.org/10.1111/codi.15056

19. Gao Y, Xi H, Chen L (2020) Emergency surgery in suspected COVID-19 patients with acute abdomen: case series and perspectives [published online ahead of print, 2020 Apr 13]. Ann Surg. https://doi.org/10.1097/SLA.0000000000003961

20. Vigneswaran Y, Prachand VN, Posner MC, Matthews JB, Hussain $M$ (2020) What is the appropriate use of laparoscopy over open procedures in the current COVID-19 climate? [published online ahead of print, 2020 Apr 13]. J Gastrointest Surg:1-6. https://doi. org/10.1007/s11605-020-04592-9

21. Chew MH, Tan WJ, Ng CY, Ng KH (2020) Deeply reconsidering elective surgery: worldwide concerns regarding colorectal surgery in a COVID-19 pandemic and a Singapore perspective [published online ahead of print, 2020 Apr 29]. Singap Med J. https://doi.org/ $10.11622 /$ smedj.2020070

22. Musa S (2020) Hepatic and gastrointestinal involvement in coronavirus disease 2019 (COVID-19): what do we know till now? Arab J Gastroenterol 21(1):3-8. https://doi.org/10.1016/j.ajg.2020.03.002

23. Chadi SA, Guidolin K, Caycedo-Marulanda A et al (2020) Current evidence for minimally invasive surgery during the COVID-19 pandemic and risk mitigation strategies: a narrative review [published online ahead of print, 2020 May 20]. Ann Surg. https://doi. org/10.1097/SLA.0000000000004010, https://doi.org/10.1097/ SLA.0000000000004010

24. Zhou F, Yu T, Du R et al (2020) Clinical course and risk factors for mortality of adult inpatients with COVID-19 in Wuhan, China: a retrospective cohort study. Lancet. https://doi.org/10.1016/S01406736(20)30566-3

25. Lei S, Jiang F, Su W et al (2020) Clinical characteristics and outcomes of patients undergoing surgeries during the incubation period of COVID-19 infection. EClinicalMedicine. 2020:100331

26. Mao R, Liang J, Shen J, Ghosh S, Zhu LR, Yang H, Wu KC, Chen $\mathrm{MH}$, Chinese Society of IBD, Chinese Elite IBD Union, Chinese IBD Quality Care Evaluation Center Committee (2020) Comment implications of COVID-19 for patients with pre-existing digestive diseases. Lancet Gastroenterol Hepatol. https://doi.org/10.1016/ S2468-1253(20)30076-5

27. Fu Y, Cheng Y, Wu Y (2020) Understanding SARS-CoV-2mediated inflammatory responses: from mechanisms to potential therapeutic tools. Virol Sin. https://doi.org/10.1007/s12250-02000207-4

Publisher's note Springer Nature remains neutral with regard to jurisdictional claims in published maps and institutional affiliations. 